\title{
Avaliação Comparativa das Propriedades de Xantanas Produzidas pelo Patovar Pruni e Clairana com Xantana Comercial para Predição de Uso
}

\author{
Patrícia D. Oliveira, Claire T. Vendruscolo \\ Centro de Desenvolvimento Tecnológico - Biotecnologia, Universidade Federal de Pelotas \\ Caroline D. Borges \\ Centro de Ciências Químicas, Farmacêuticas e de Alimentos, Universidade Federal de Pelotas \\ Ricardo C. Michel \\ Instituto de Macromoléculas Professora Eloísa Mano, Universidade Federal do Rio de Janeiro \\ Rosana T. Lomba \\ Tecnologia de Engenharia de Poço, Centro de Pesquisas e Desenvolvimento Leopoldo \\ Américo Miguez de Mello, Petrobras
}

\begin{abstract}
Resumo: $O$ presente trabalho teve como objetivo determinar a adequabilidade de três amostras de biopolímero xantana (Xa 06, Xa 82 e Xa 106) e uma clairana como viscosificantes de fluidos de perfuração de poços de petróleo, comparando com uma amostra de xantana comercial, Xanvis ${ }^{\circledast}$. Foram realizadas análises reológicas, determinados os conteúdos de acetil, piruvato e íons $\mathrm{Na}^{+} \mathrm{K}^{+}$e $\mathrm{Ca}^{2+}$, e avaliada a capacidade viscosificante segundo norma específica da Petrobras. Os conteúdos de grupos acetil e piruvato para as xantanas e clairana diferiram significativamente entre si, mas foram compatíveis com valores preconizados ou citados na literatura concernente. Com relação ao conteúdo de íons, a xantana comercial apresentou quantidade de cálcio superior, as amostras Xa 06, Xa 82 e Xa 106 tiveram maior teor de potássio e a clairana maior teor de sódio. A viscosidade e a viscoelasticidade da amostra de xantana comercial foram superiores às demais amostras analisadas. $\mathrm{Na}$ análise como viscosificante para fluidos de perfuração as amostras de xantana comercial e Xa 106 atingiram os requisitos estabelecidos para os parâmetros n, K e força gel da norma Petrobras N-2605. Com a interpretação conjunta dos resultados obtidos pode-se inferir que apenas as xantanas Xanvis ${ }^{\circledR}$ e Xa 106 apresentam características adequadas à utilização como viscosificante em fluido de perfuração de petróleo. As demais amostras possuem características reológicas adequadas à utilização como espessantes ou estabilizantes em outros segmentos industriais, como em alimentos, tintas e cosméticos.
\end{abstract}

Palavras-chave: Biopolímeros, viscosidade, acetil, piruvato, íons.

\section{Comparative Evaluation of Xanthans Properties Produced by Pathovar Pruni and Clairana with Commercial Xanthan to Predict their Uses}

\begin{abstract}
The aim of this study was to determine the suitability of three xanthans (Xa 06, Xa 82 and Xa 106) and a clairana biopolymer for use as drilling fluid thickeners and comparing them against a commercial sample, Xanvis ${ }^{\circledR}$. Rheological analyses were performed to determine the acetyl, pyruvate and $\mathrm{Na}^{+} \mathrm{K}^{+}$and $\mathrm{Ca}^{2+}$ ion content and their thickening ability, according to Petrobras guidelines. The pyruvate and acetyl group content for the xanthans and the clairana biopolymer differed significantly, but they were consistent with the recommended values or those previously cited in the literature. With regard to the ion content, the commercial xanthan contained higher amounts of calcium, while Xa 06, Xa 82 and Xa 106 samples contained higher potassium levels and clairana had a higher sodium level. The viscosity and viscoelasticity of the commercial xanthan sample were higher than for the other samples. In the viscosity analysis of drilling fluids, Xanvis ${ }^{\circledR}$ and Xa 06 met the required parameters for n, K and gel strength (Petrobras regulation $\mathrm{N}-2605)$. In an analysis of the results obtained, only Xanvis ${ }^{\circledR}$ and Xa 106 presented the desired characteristics for use as drilling fluid thickeners. The other samples presented adequate rheological characteristics for use as thickeners and stabilizers in many industrial applications, such as food, paints and cosmetics.
\end{abstract}

Keywords: Biopolymers, viscosity, acetyl, pyruvate, ions. 


\section{Introdução}

Existe um grande interesse industrial pelos polissacarídeos microbianos extracelulares como xantana, pululana e dextrana que, por suas propriedades, possuem uma vasta aplicabilidade como viscosificante, gelificantes e estabilizantes em diversos setores industriais. Devido a esse interesse, estes biopolímeros, no Brasil, foram incluídos nos estudos "Biopolímeros e Intermediários Químicos do Centro de Gestão e Estudos Estratégico"[1] e "Estratégia Nacional de Biotecnologia - Política de Desenvolvimento da Bioindústria do Ministério de Desenvolvimento da Indústria e Comércio"[2], os quais serviram de subsídios para elaboração da Política de Biotecnologia Industrial no Brasil de 2006 a 2011.

O grande interesse industrial nos polissacarídeos microbianos se deve às suas propriedades físicas e químicas únicas, que os tornam superiores a outros polissacarídeos $^{[3]}$. No entanto, uma combinação de propriedades é fundamental para definir sua aplicação final, e estas são determinadas pela composição química, ligações intra e intermoleculares, massa molar e sua distribuição, além dos teores de acetil, piruvato e íons presentes na molécula. Estudos de muitos biopolímeros têm sido relatados na literatura pertinente, os quais objetivam o conhecimento da estrutura química e do comportamento das suas propriedades em solução ${ }^{[3-6]}$. Para se obter novos biopolímeros microbianos para aplicação industrial, cepas das bactérias Xanthomonas arboricola pv pruni e Beijerinckia sp têm sido selecionadas quanto a sua produtividade e os biopolímeros obtidos analisados quanto a viscosidade e composição química ${ }^{[7-16]}$.

$\mathrm{O}$ presente trabalho teve como objetivo determinar a adequabilidade de três amostras de biopolímero xantana, sintetizados por cepas da bactéria $X$. arboricola pv pruni, e uma de clairana, produzida por Beijerinckia sp, como viscosificantes de fluidos de perfuração de poços de petróleo, comparando com uma amostra de xantana comercial, Xanvis ${ }^{\circledR}$.

\section{Materiais e Métodos}

\section{Produção dos biopolímeros}

As amostras de xantana foram sintetizadas pela bactéria Xanthomonas arboricola pv pruni cepas 06, 82 e 106, as quais neste trabalho serão denominadas de Xa 06, Xa 82 e Xa 106. A amostra de clairana, constituída de fibras longas, foi sintetizada pela bactéria Beijerinckia sp. cepa 7070, isolada de solo canavieiro por Vendruscolo ${ }^{[7]}$.

Os meios de cultura bem como os processos, são os descritos na invenção WO 2006/047845 A1 - Xanthan biopolymer, process and uses of the same ${ }^{[17]}$ e PI 01058568 - Processo de produção de polissacarídeo por Beijerinckia $\mathrm{sp}^{[18]}$. Os inóculos foram produzidos em incubador agitador orbital (New Brunswick ${ }^{\circledR}$, Scientific, modelo Innova 4230) e as fermentações conduzidas em biorreator de bancada (B. Braun Biotech ${ }^{\circledR}$. Inc., modelo Biostat B), utilizando vaso de 10L. Os polímeros recuperados foram secos em estufa a $56{ }^{\circ} \mathrm{C}$ e triturados em moinho de disco (Fritsch ${ }^{\circledR}$, Pulverisette).

Como padrão utilizou-se a xantana comercial de grau industrial Xanvis ${ }^{\circledR}$, produzida pela empresa Kelco ${ }^{\circledR}$ (Houston - Estados Unidos da América), fornecida pelo Laboratório de Fluidos do Centro de Pesquisas e Desenvolvimento (Cenpes) da Petrobras.

\section{Determinação dos teores de acetil e piruvato}

A quantificação dos ácidos acético e pirúvico foi realizada por colorimetria, utilizando os métodos do ácido hidroxâmico ${ }^{[19]}$ e da 2,4-dinitrofenilhidrazinaa ${ }^{[20]}$, respectivamente. A análise estatística foi realizada com o programa Statistics (8.0) com análise de variância (ANOVA) seguida do teste de Tukey, com nível de significância de $5 \%$.

\section{Determinação dos teores de sódio, potássio e cálcio}

As quantificações dos íons cálcio, sódio e potássio das amostras foram realizadas por espectrometria de emissão atômica utilizando fotômetro de chama (Cole Parmer ${ }^{\circledR}$, modelo 2655-00). Os íons foram mensurados segundo metodologia clássica ASTM D1428-64 ${ }^{[21]}$.

\section{Análises reológicas}

As amostras foram preparadas em água destilada com $1 \times 10^{-3} \mathrm{~g} . \mathrm{L}^{-1}$ de azida sódica, sob agitação magnética com aquecimento a $40{ }^{\circ} \mathrm{C}$ por 16 horas. A concentração das soluções foi de 5,0 g. $\mathrm{L}^{-1}$ e verificada por gravimetria. As análises foram realizadas em reômetro Rheostress $600\left(\right.$ Haake $\left.^{\circledR}\right)$ com controlador de temperatura Peltier ${ }^{\circledR}$ $\left( \pm 0,1^{\circ} \mathrm{C}\right)$ nas temperaturas de $25^{\circ} \mathrm{C}, 45^{\circ} \mathrm{C}$ e $65^{\circ} \mathrm{C}$. Para retardar a evaporação da água nas temperaturas acima de $25^{\circ} \mathrm{C}$, uma fina camada de óleo newtoniano foi utilizada ao redor do cone, após o gap ter sido atingido, recobrindo a superfície da amostra exposta. A viscosidade foi medida com a taxa de cisalhamento variando de 1 a $1000 \mathrm{~s}^{-1}$. As medidas oscilatórias foram determinadas com frequência variando de $0,02-10 \mathrm{~Hz}$ na região de viscoelasticidade linear em taxa de deformação de $0,05 \mathrm{~Pa}$. A geometria utilizada foi cone e placa (diâmetro $60,0 \mathrm{~mm}$; distância 0,105 mm; ângulo $2^{\circ}$ ).

\section{Capacidade viscosificante em fluido de perfuração de poços de petróleo}

Preparou-se soluções aquosas de acordo com a seguinte composição: 60 g.L. ${ }^{-1}$ de NaCl, 0,2 g.L $L^{-1}$ de $\mathrm{CaCl}_{2}$, 0,06 g.L $\mathrm{L}^{-1}$ de $\mathrm{MgCl}_{2}$ e 4,3 g.L $\mathrm{L}^{-1}$ de xantana. O método de preparo seguiu as especificações contidas na norma N-2605 da Petrobras ${ }^{[22]}$. As análises foram realizadas em reômetro Rheostress 100 (Haake ${ }^{\circledR}$ ) com controlador de temperatura Peltier ${ }^{\circledR}\left( \pm 0,1{ }^{\circ} \mathrm{C}\right)$. A variação da taxa de cisalhamento foi de $1 \mathrm{a} 1000 \mathrm{~s}^{-1}$ e a geometria utilizada foi cone e placa. As análises de força gel foram realizadas em viscosímetro Fann ${ }^{\circledR}$ modelo $35 \mathrm{~A}$, utilizando bob com raio de $1,72 \times 10^{-2} \mathrm{~m}$, na temperatura de $25^{\circ} \mathrm{C}$. 


\section{Resultados e Discussões}

\section{Teores de acetil e piruvato}

Os conteúdos de acetil e piruvato dos biopolímeros são apresentados na Tabela 1.

Os teores de acetil e piruvato diferiram significativamente entre todas as amostras $(\mathrm{p}<0,05)$, inclusive para as xantanas produzidas pelo mesmo patovar, indicando que estes constituintes são cepas dependentes. No entanto, todos os percentuais de piruvato encontram-se acima do valor mínimo de 1,5\% preconizado para xantanas comerciais ${ }^{[23,24]}$ e dentro da faixa registrada na literatura para amostras de xantana (Tabela 2), que é de 0,24 a 6,0\%. Não existe uma regulamentação para valores mínimos de acetil, mas os resultados obtidos para as xantanas analisadas encontram-se dentro da variação registrada na literatura, a qual está compreendida entre 0,2 e 14,3\%, (Tabela 2). $\mathrm{O}$ maior grau de acetil e piruvato nas amostras de xantana foi verificado para a Xanvis ${ }^{\circledR}$, e o menor na Xa 106. É importante observar que a maioria das amostras de xantana apresentou quantidade estequiométrica entre os grupos acetil e piruvato. Comportamento semelhante foi observado por Bradshaw et al. ${ }^{[5]}$ para xantana comercial Keltrol $^{\circledR}$ e por Shatwell et al. ${ }^{[25]}$ para a xantana sintetizada por X. campestris pv campestris cepa 646. Entretanto, a maior parte dos resultados publicados não apresenta quantidade estequiométrica entre estes grupos, sendo o grau de substituição por acetil superior ao por piruvato, como verificado por Teixeira ${ }^{[12]}$ e Pinto $^{[13]}$ (Tabela 2). Estes autores, embora tenham trabalhado com o mesmo patovar do presente estudo, obtiveram valores diferentes, visto terem utilizado cepas diferentes (com exceção da cepa 06, também utilizada em nosso estudo e por Teixeira ${ }^{[12]}$ ), bem como escala de produção, que foi menor, realizada em Erlenmeyer, em volume de 0,1 L.

O valor encontrado para piruvato na clairana é compatível com o preconizado para xantana comercial ${ }^{[23,24]}$; já o teor de acetil encontra-se dentro da faixa relatada na literatura (Tabela 2). A xantana é o polissacarídeo aniônico mais amplamente estudado, diferentemente da clairana, sobre a qual, por ser um biopolímero relativamente novo, dispõem-se de poucos dados. As pesquisas referentes à composição química e estrutura da clairana, realizadas anteriormente, não relataram a presença desses grupos na molécula ${ }^{[26]}$. Porém, Ohtani et al. ${ }^{[27]}$ estudaram a caracterização do polissacarídeo sintetizado por Beijerinckia indica cepa TX-1 e detectaram teor de $16,2 \%$ de acetil na estrutura, valor muito superior ao determinado no presente estudo. Cooke e Percival ${ }^{[28]}$, no polissacarídeo extracelular sintetizado por Beijerinckia mobilis cepa NBC 9879, através da mesma metodologia utilizada neste trabalho, determinaram teor de 2,6\% de piruvato na estrutura do biopolímero como substituinte ácido, valor próximo ao verificado neste trabalho.

Tem sido demonstrado que o grau de substituição por acetil e piruvato na xantana tem influência sobre a estabilidade da estrutura na forma de hélice e sobre a faixa de temperatura em que ocorre a transição conformacional da forma estruturada para enovelamento ao acaso ${ }^{[2,30]}$, bem como na capacidade de associação intermolecular ${ }^{[31]}$ e intramolecular ${ }^{[32]}$; sendo que todos estes fatores interferem na capacidade viscosificante do biopolímero $^{[25]}$.

Tabela 1. Teores de acetil e piruvato das xantanas e clairana.

\begin{tabular}{lccccc}
\hline & \multicolumn{5}{c}{ Amostra } \\
\cline { 2 - 6 } & Xa 06 & Xa 82 & Xa 106 & Xanvis $^{\otimes}$ & clairana \\
\hline Acetil (\%) & $2,76 \pm 0,01$ & $3,29 \pm 0,01$ & $2,56 \pm 0,01$ & $3,88 \pm 0,01$ & $3,37 \pm 0,01$ \\
Piruvato (\%) & $2,48 \pm 0,06$ & $3,34 \pm 0,01$ & $0,78 \pm 0,04$ & $3,90 \pm 0,06$ & $1,96 \pm 0,05$ \\
\hline
\end{tabular}

*Médias de três análises \pm desvio padrão.

Tabela 2. Teores de acetil e piruvato para diferentes xantanas citados na literatura.

\begin{tabular}{|c|c|c|c|}
\hline Referência & Amostra & Acetil $(\%)$ & Piruvato $(\%)$ \\
\hline$[20]$ & X. campestris pv campestris (NRRL B-1459) & 4,6 & --- \\
\hline$[5]$ & Keltrol $^{\circledR}($ Kelco $)$ & 4,1 & 4,3 \\
\hline \multirow{4}{*}[25]{} & X. campestris pv campestris 646 & 4,5 & 4,4 \\
\hline & X. campestris pv phaseoli 1128 & 7,7 & 1,7 \\
\hline & X. campestris pv oryzae PXO61 & 14,3 & 0,3 \\
\hline & X. campestris pv phaseoli 556 & 1,6 & 6,0 \\
\hline \multirow{3}{*}{ [29] } & $X$. campestris pv campestris & 1,0 & 0,7 \\
\hline & X. campestris pv gummisudans 2182 & 1,5 & 0,7 \\
\hline & X. campestris pv campestris BD9A & 0,2 & 0,1 \\
\hline$[30]$ & Kelco & 5,14 & 0,24 \\
\hline \multirow{2}{*}[12]{} & X. arboricola pv pruni 101 & 5,42 & 0,26 \\
\hline & $X$. arboricola pv pruni 06 & 3,25 & 2,81 \\
\hline \multirow{2}{*}{ [13] } & Jungbunzlauer & 4,1 & 3,6 \\
\hline & X. arboricola $\mathrm{pv}$ pruni 101 & 5,0 & 0,8 \\
\hline [15] & X. arboricola $\mathrm{pv}$ pruni 106 & 3,5 & 0,31 \\
\hline
\end{tabular}




\section{Teores de sódio, potássio e cálcio}

Nos processos fermentativos utilizam-se substratos constituídos por fonte de carbono, nitrogênio e sais minerais, os quais são adicionados em quantidades que normalmente excedem o consumo pelas bactérias. Os cátions destes sais ligam-se aos radicais aniônicos dos substituintes acetil e piruvato presentes na xantana e clairana. Os teores dos cátions verificados nas amostras são apresentados na Tabela 3.

Os teores dos íons diferiram entre as amostras. Observou-se que a quantidade de cálcio presente na Xanvis $^{\circledR}$ é muito superior a quantidade presente nos demais biopolímeros. Este alto teor de cálcio foi relatado por Ferreira et al. ${ }^{[33]}$ e por Borges et al. ${ }^{[15]}$ para amostras comerciais. García-Ochôa et al. ${ }^{[34]}$, entretanto, relatam em sua revisão, valores entre 0,085 e $0,17 \%(\mathrm{~m} / \mathrm{m})$ para cátions bivalentes e entre 3,6 e $14,3 \%(\mathrm{~m} / \mathrm{m})$ para cátions monovalentes. As xantanas Xa 06 e Xa 82 apresentaram maior teor de potássio, enquanto a Xa 106 apresentou o menor teor, inclusive inferior ao da Xanvis ${ }^{\circledR}$. Teores de sódio e potássio superiores foram determinados por Pinto ${ }^{[13]}$ para uma xantana comercial $\left(28,2\right.$ g. $\mathrm{L}^{-1}$ de sódio e 1,98 g.L $\mathrm{L}^{-1}$ de potássio). Já para a xantana sintetizada por $X$. arboricola pv pruni cepa 101, detectou 2,02 g. $\mathrm{L}^{-1}$ de sódio e 1,98 g.L. ${ }^{-1}$ de potássio. Para Xa 101 e Xa 106, teores de sódio muito superiores aos de potássio são relatados ${ }^{[15]}$. Este fato foi atribuído à utilização de hidróxido de sódio para o controle do $\mathrm{pH}$ durante a fermentação. Como as fermentações do presente trabalho foram realizadas sem controle de $\mathrm{pH}$, pode-se afirmar que a quantidade de íons presentes em amostras de xantana é variável e dependente das cepas e também dos processos fermentativos.

$\mathrm{Na}$ clairana verificou-se teores de sódio e potássio muito acima dos valores relatados para xantana de modo geral; já o cálcio foi igual ao apresentado pelas xantanas do patovar pruni. Uma super concentração de sais pode afetar negativamente as características estruturais e as propriedades reológicas do polieletrólito xantana, porém a intensidade do efeito é variável em função de características específicas de cada xantana ${ }^{[35]}$. As elevadas concentrações de íons verificadas na clairana podem, semelhantemente, estarem afetando negativamente sua viscosidade.

\section{Análises reológicas}

Os resultados das análises reológicas dos biopolímeros são apresentados na Figura 1. Os biopolímeros tiveram comportamento pseudoplástico, como esperado para soluções aquosas de $x^{2} \operatorname{santana}^{[36,8]}$ e para soluções de clairana ${ }^{[14]}$. A clairana, entretanto, em taxas de cisalhamento abaixo de $10 \mathrm{~s}^{-1}$, apresentou, conforme a temperatura da análise, reduzida pseudoplasticidade ou comportamento newtoniano. As xantanas Xa 06 e Xa 106 apresentaram comportamento similar ao da Xanvis ${ }^{\circledR}$, com relação à pseudoplasticidade. A Xa 82 e a clairana apresentaram menor pseudoplasticidade em baixas taxas de cisalhamento, caracterizada por menor decréscimo da viscosidade em relação à Xanvis ${ }^{\circledR}$. Com o aumento da temperatura ocorreu redução da viscosidade e pseudoplasticidade. Possivelmente, este comportamento possa ser atribuído a uma maior flexibilidade das cadeias dos biopolímeros Xa 82 e clairana. Em baixas taxas de cisalhamento, a Xa 106 e a Xa 06 apresentaram viscosidades semelhantes, menores que o resultado da Xanvis ${ }^{\circledast}$, e superiores às demais amostras, em todas as temperaturas. Todos os biopolímeros apresentaram decréscimo da viscosidade com o aumento da temperatura. Porém, a Xa 06, Xa 106 e a Xanvis ${ }^{\circledR}$ foram mais estáveis frente à variação da temperatura, enquanto a Xa 82 apresentou o maior decréscimo.

Não foi verificado no estudo relação direta entre teor de piruvato e viscosidade. Verificou-se, na Xanvis ${ }^{\circledR}$, teores de piruvato muito semelhantes ao da Xa 82, porém a sua viscosidade foi bem maior; já a Xa 06, com teores inferiores a $\mathrm{Xa} 82$, teve uma viscosidade maior do que esta. A Xa 106, com a segunda maior viscosidade, teve o menor teor de piruvato entre todas as amostras de xantana. Não existe um consenso na literatura sobre a influência exercida pelo substituinte piruvato sobre a viscosidade da xantana. Para Sandford et al. ${ }^{[36]}$ e Cadmus et al. ${ }^{[37]}$, o aumento no teor de piruvato correspondeu a aumento da viscosidade. Por outro lado, alguns estudos mostraram baixa ou nenhuma correlação entre o teor de piruvato e a viscosidade ${ }^{[5,15,25,32]}$. Quanto aos teores de acetil, de modo geral tem-se relacionado à desacetilação ou redução dos teores com o aumento da viscosidade ${ }^{[3,13,25]}$. Neste estudo, as xantanas do patovar pruni com menores teores de acetil tiveram maiores viscosidades, enquanto que a Xanvis ${ }^{\circledR}$, para a qual detectou-se o maior teor de acetil, teve a maior viscosidade. Entretanto, isto pode ser explicado por outros fatores.

Conforme Silveira et al. ${ }^{[38]}$ vários componentes, como acetil, piruvato, íons e massa molar, influenciam conjuntamente à viscosidade das xantanas. Acertadamente, segue-se que, para definir a aplicação de um biopolímero, é necessária uma combinação de propriedades. A viscosidade superior da Xanvis ${ }^{\circledR}$ em relação às demais amostras, possivelmente seja devida ao maior teor de cálcio (Tabela 3). A presença de íons divalentes, como o cálcio, favorece a formação de aglomerados entre as moléculas de xantana e auxilia na estabilidade frente a variações de temperatura e cisalhamento ${ }^{[31]}$. Por isso, normalmente, a xantana utilizada em fluidos de perfuração de poços, como a Xanvis ${ }^{\circledR}$, contém aditivos, como carbonato de cálcio, para melhorar/estabilizar suas propriedades reológicas ${ }^{[39]}$.

Tabela 3. Teores de sódio, potássio e cálcio determinados nas xantanas e clairana.

\begin{tabular}{cccccc}
\hline \multirow{2}{*}{ Cátions $\left(\mathbf{g . L} \mathbf{L}^{-1}\right)$} & \multicolumn{5}{c}{ Amostra } \\
\cline { 2 - 6 } & Xa 06 & Xa 82 & Xa 106 & Xanvis $^{\circledast \text { c }}$ & clairana \\
\hline $\mathrm{Na}^{+}$ & 5,40 & 1,00 & 1,66 & 0,26 & 98,0 \\
$\mathrm{~K}^{+}$ & 11,0 & 13,0 & 0,35 & 2,70 & 38,0 \\
$\mathrm{Ca}^{2+}$ & 0,05 & 0,06 & 0,05 & 2,80 & 0,05 \\
\hline
\end{tabular}


Os resultados apresentados na Figura 2 mostram o comportamento dos módulos elástico e viscoso em função da frequência, nas diferentes temperaturas testadas. Todos os biopolímeros apresentaram diminuição no valor dos módulos com o aumento da temperatura.

Para a Xanvis ${ }^{\circledR}$ e Xa 106, Figura 2a, d, o módulo elástico (G') foi, em geral, maior que o módulo viscoso $\left(G^{\prime \prime}\right)$, mostrando a existência de estrutura de gel. Os módulos apresentam sobreposição somente em baixas frequências para a temperatura de $65^{\circ} \mathrm{C}$. Para a Xa 06, Figura 2b, o módulo elástico (G') apresentou-se superior ao viscoso (G") a partir da frequência de $0,07 \mathrm{~Hz}$ em $25^{\circ} \mathrm{C}$; a partir de $0,17 \mathrm{~Hz}$ em $45^{\circ} \mathrm{C}$ e de $1,67 \mathrm{~Hz}$ quando em $65^{\circ} \mathrm{C}$. Comportamento semelhante foi verificado na Xa 82 a partir da frequência de $0,2 \mathrm{~Hz}$ em $25^{\circ} \mathrm{C}, 0,6 \mathrm{~Hz}$ em $45^{\circ} \mathrm{C}$ e $0,07 \mathrm{~Hz}$ em $65^{\circ} \mathrm{C}$.

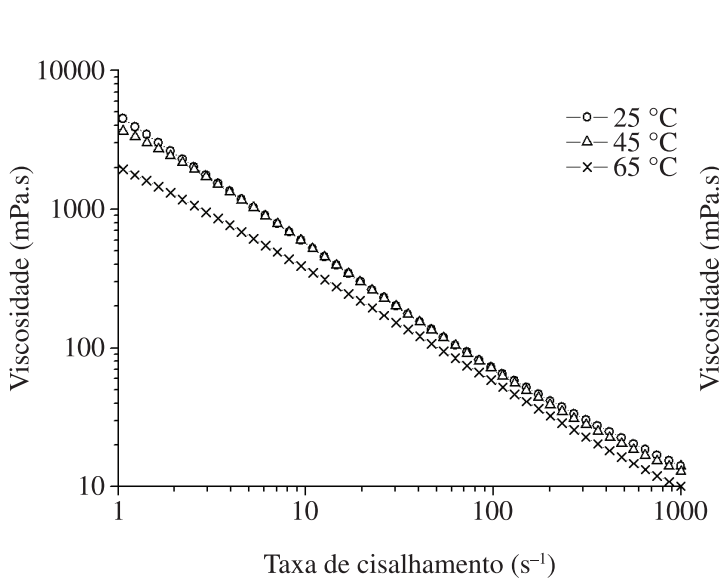

(a)

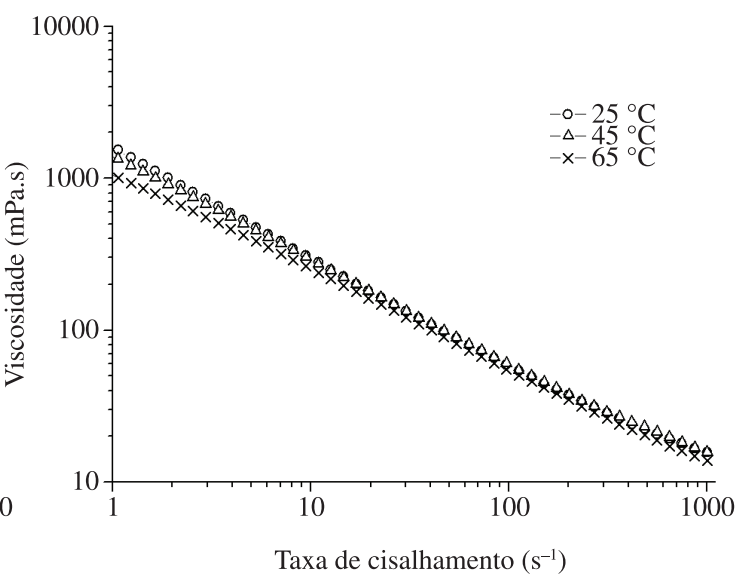

(b)

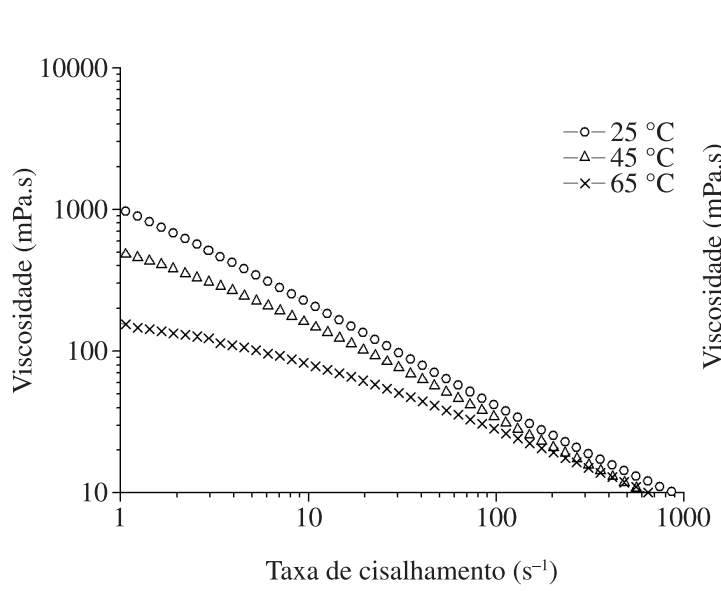

(c)

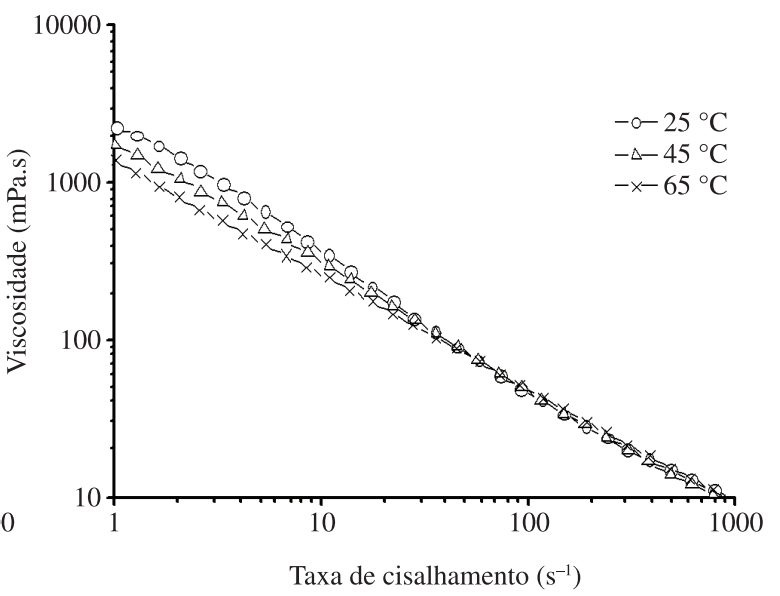

(d)

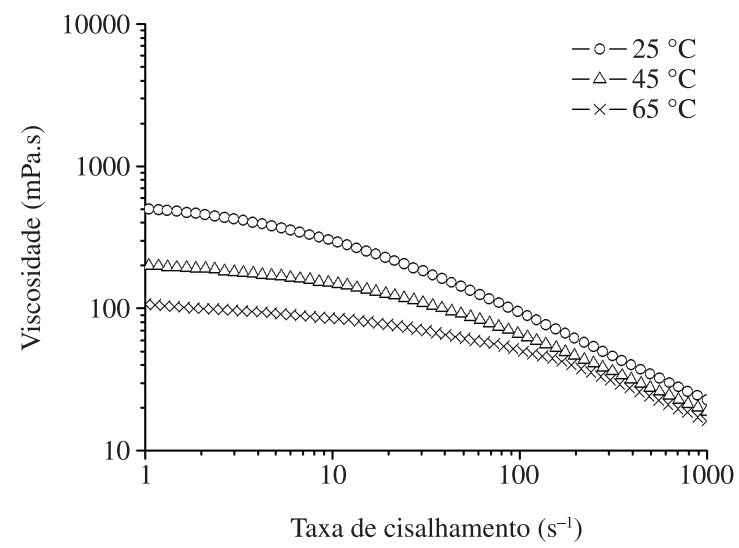

(e)

Figura 1. Curvas de viscosidade para as soluções aquosas dos biopolímeros (a) Xanvis ${ }^{\circledR}$, (b) Xa 06 (c) Xa 82 (d) Xa 106 e (e) clairana, nas temperaturas de $25^{\circ} \mathrm{C}, 45^{\circ} \mathrm{C}$ e $65^{\circ} \mathrm{C}$. 
Nas curvas relativas à clairana, Figura 2e, observa-se módulo viscoso superior ao elástico em todas as temperaturas analisadas, mostrando não haver formação de gel, nas condições estudadas. Comportamento similar foi obtido por Martínez-Padilla e Cabada-Celis ${ }^{[40]}$. Porém, Boza et al. ${ }^{[41]}$ verificaram ocorrência de resposta elástica para a clairana, em solução com concentração de 30,0 g. $\mathrm{L}^{-1}$, em frequência superior a $3,5 \mathrm{~Hz}$, sendo independente da frequência a partir desse ponto.

\section{Capacidade viscosificante em fluido de perfuração de poços}

$\mathrm{Na}$ Petrobras a capacidade viscosificante dos polímeros utilizados em fluidos de perfuração de poços é analisada segundo a Norma Técnica N-2605 A, sendo os mesmos solubilizados em meio salino e analisados quanto aos parâmetros $\mathrm{K}$ e n, do modelo de Ostwald-deWaelle, e quanto as suas propriedades de gel ${ }^{[22]}$.

As curvas de viscosidade obtidas para as xantanas são apresentadas na Figura 3. As xantanas do patovar pruni

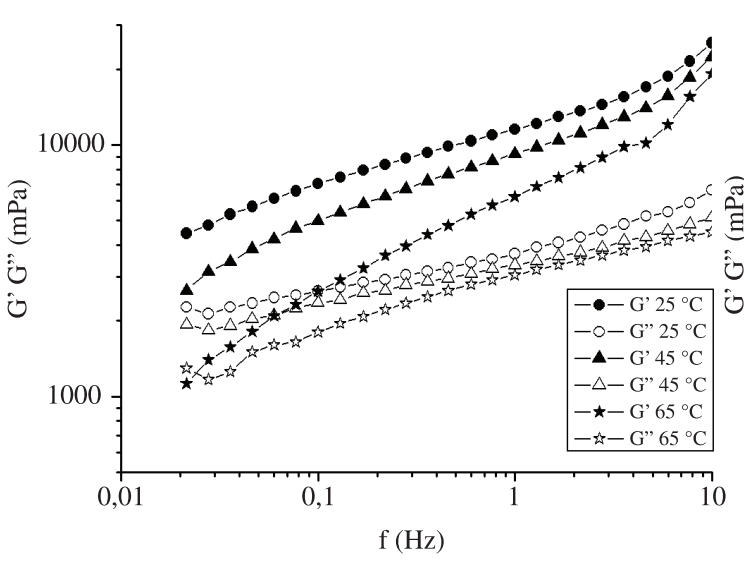

(a)

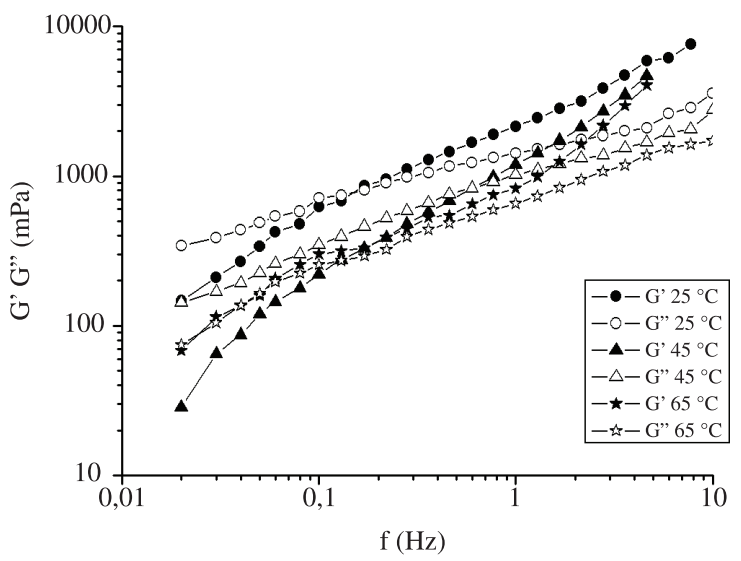

(c)

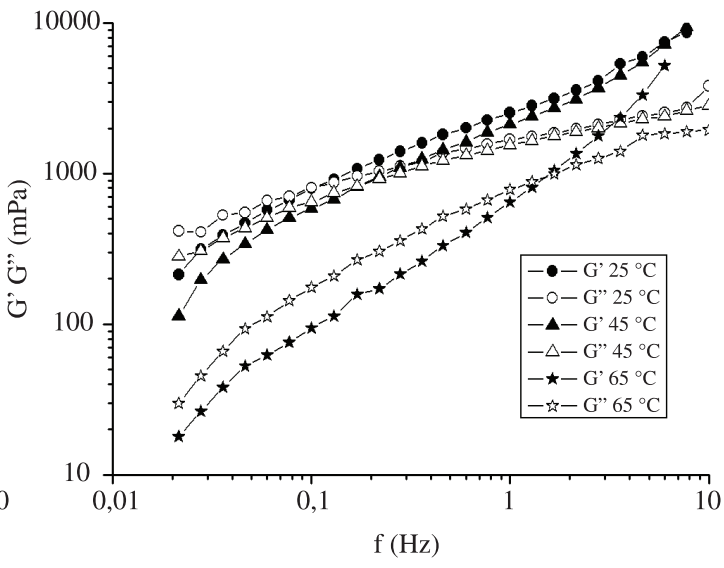

(b)

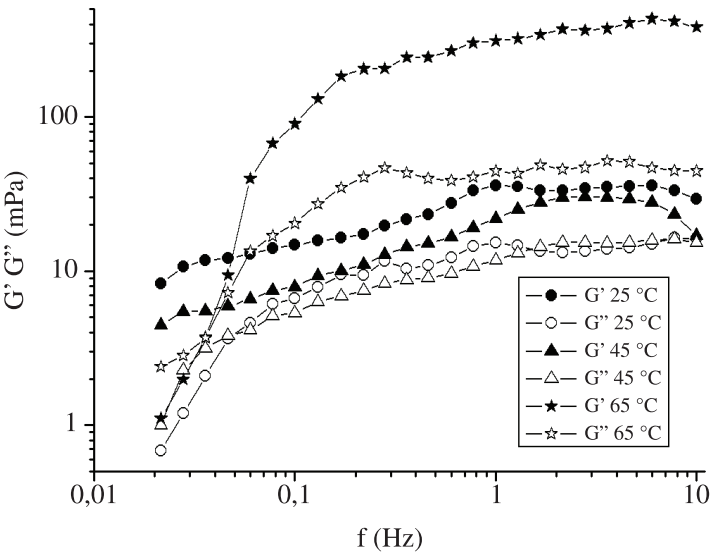

(d)

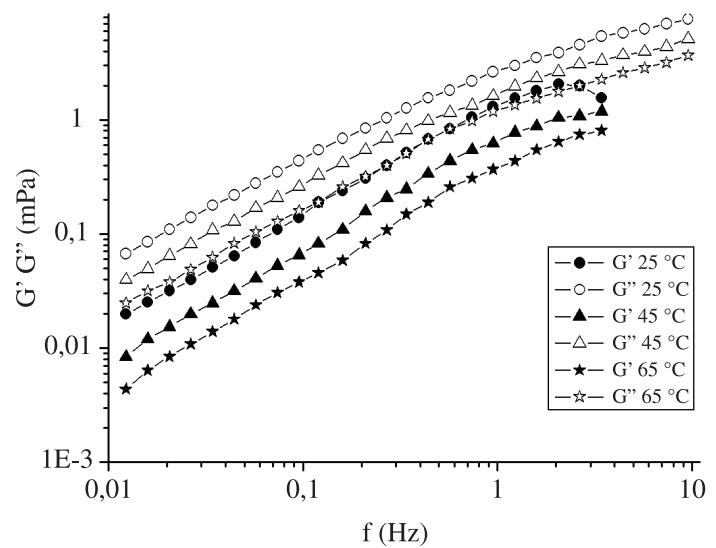

(e)

Figura 2. Curvas dos módulos elástico e viscoso das soluções aquosas de (a) Xanvis ${ }^{\circledR}$, (b) Xa 06, (c) Xa 82, (d) Xa 106 e (e) clairana, nas temperaturas de $25^{\circ} \mathrm{C}, 45^{\circ} \mathrm{C}$ e $65^{\circ} \mathrm{C}$. 


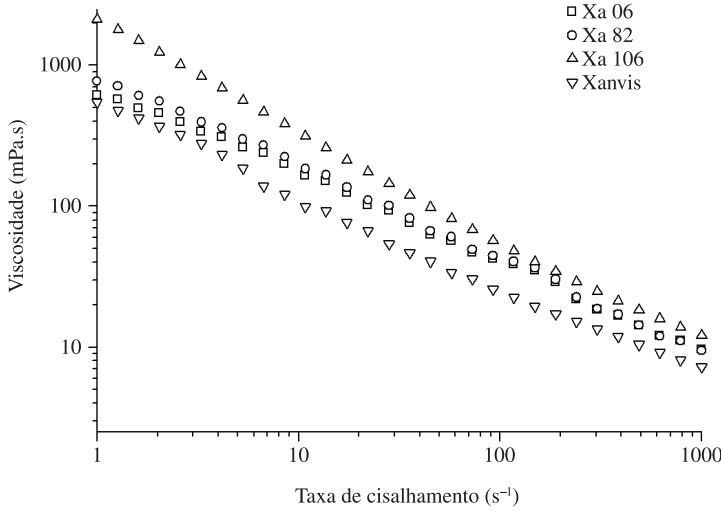

Figura 3. Curvas de viscosidade para as soluções salinas dos biopolímeros.

Tabela 4. Resultados dos parâmetros n e K das amostras de xantana em solução salina.

\begin{tabular}{|c|c|c|c|c|c|}
\hline \multirow{2}{*}{$\begin{array}{l}\text { Amostra } \\
\text { Xa } 06\end{array}$} & \multirow{2}{*}{$\begin{array}{c}\mathbf{n}(\mathbf{a d m}) \\
0,318\end{array}$} & \multirow{2}{*}{$\begin{array}{c}\begin{array}{c}\text { K } \\
\text { (mPa.s) }\end{array} \\
1597,44\end{array}$} & \multicolumn{3}{|c|}{$\begin{array}{c}\text { Força gel }\left(\mathrm{lbf} / 100 t^{2}\right) \\
\qquad G_{0} G_{10} G_{30}\end{array}$} \\
\hline & & & 5 & 5 & 6 \\
\hline Хa 82 & 0,468 & 965,18 & 4 & 5 & 5 \\
\hline Ха 106 & 0,204 & 2140,00 & 9 & 13 & 14 \\
\hline Xanvis $^{\circledast}$ & 0,275 & 1590,05 & 12 & 15 & 16 \\
\hline
\end{tabular}

apresentaram viscosidade superior à da Xanvis ${ }^{\circledR}$, sendo a Xa 106 a mais viscosa. Contrário ao comportamento apresentado em solução aquosa, a Xa 82 em solução salina apresentou viscosidade similar àquela da Xa 06. Os resultados demonstram a maior compatibilidade das xantanas do patovar pruni em soluções salinas quando comparados à Xanvis ${ }^{\circledR}$.

A norma $\mathrm{N}-2604^{[42]}$ especifica que o índice de consistência (K) mínimo deve ser de $1500 \mathrm{mPa} . \mathrm{s}$ e o índice de fluxo (n) deve apresentar valor máximo de 0,5. Comparando os resultados dos parâmetros $\mathrm{K}$ e $\mathrm{n}$ (Tabela 4), observa-se que a Xa 06, Xa 106 e a Xanvis ${ }^{\circledR}$ estão em conformidade com os valores estipulados na norma citada. Xa 06 e a Xanvis ${ }^{\circledR}$ foram muito similares, enquanto que a Xa 106 apresentou $\mathrm{K}$ superior ao da Xanvis $^{\circledR}$ e $\mathrm{n}$ semelhante. Portanto, estas três xantanas podem ter a mesma aplicação no que diz respeito a este quesito. Já a amostra Xa 82 não atingiu as especificações da norma. Para a clairana não foram determinados os índice $\mathrm{K}$ e $\mathrm{n}$ por sua viscosidade inferior.

A força gel é um parâmetro utilizado para indicar o grau de gelificação. A força gel inicial $\left(\mathrm{G}_{0}\right)$ mede a resistência inicial ao fluxo do fluido e as forças géis $\mathrm{G}_{10}$ e $\mathrm{G}_{30}$ medem a resistência ao reinicio do fluxo quando o fluido permanece por 10 e 30 minutos em repouso, respectivamente. A norma N-2604 ${ }^{[42]}$ estabelece (em lbf/100ft ${ }^{2}$ ) que o valor mínimo para $\mathrm{G}_{0}$ é de 7 ; para $G_{10}$ entre 10 e 15 e $G_{30}$ valor máximo de 20 . Os resultados obtidos para estes índices são apresentados na Tabela 4. Nas análises de força gel, apenas a Xanvis ${ }^{\circledR}$ e a Xa 106 apresentaram valores de acordo com os especificados pela norma $\mathrm{N}-2604^{[42]}$, sendo os demais valores inferiores aos considerados como mínimos pela norma. As demais xantanas e clairana podem ser utilizadas como espessantes em várias outras áreas, como na indústria de tintas, cosméticos e etc., onde os índices $n$ e $\mathrm{K}$ e os módulos viscoso e elástico não são exigidos ${ }^{[23]}$. Entretanto, somente a xantana tem o uso aprovado em alimentos $^{[43]}$

\section{Conclusão}

Em todas as amostras analisadas verificaram-se concentrações de piruvato e acetil em acordo com o preconizado ou relatado para xantanas comerciais. As amostras tiveram concentrações de íons monovalentes semelhantes às relatadas na literatura para xantana, com exceção da clairana, que teve valores superiores. A elevada concentração de cálcio verificada na Xanvis $^{\circledR}$ provavelmente contribuiu para sua viscosidade superior. Todas as amostras tiveram comportamento pseudoplástico, com viscosidade variada. Nas soluções de Xanvis $^{\circledR}$ e de Xa 106, em determinadas condições, pôde ser detectado comportamento viscoelástico de gel verdadeiro. Apenas a Xanvis ${ }^{\circledR}$ e a Xa 106 atingiram os requisitos estipulados pela norma N-2604 da Petrobras para viscosificantes em fluido de perfuração de poços.

\section{Referências Bibliográficas}

1. Brasil. Ministério do Desenvolvimento, Indústria e Comércio Exterior. Centro de Gestão e Estudos Estratégicos - CGEE. "Relatório Técnico no 84 396-205", CGEE "2006”. Disponível em: <http://www.anbio.org.br/ pdf/2/tr06_biopolimeros.pdf $>$.

2. Brasil. Ministério do Desenvolvimento,Indústriae Comércio Exterior. Secretaria do Desenvolvimento da Produção. Fórum de Competitividade de Biotecnologia. - "Estratégia Nacional de Biotecnologia - Política de Desenvolvimento da Bioindústria”, MDIC (2006). Disponível em: <http:// www.uni-muenster.de>.

3. Villain-Simonnet, A.; Milas, M. \& Rinaudo, M. - Int. J. Biol. Macromol., 26, p.55 (1999). http://dx.doi.org/10.1016/ S0141-8130(99)00062-8

4. Jansson, P. E.; Kenne, L. \& Lindberg, B. - Carbo. Res., 45, p.275 (1975). http://dx.doi.org/10.1016/S00086215(00)85885-1

5. Bradshaw, I. J.; Nisbet, B. A.; Kerr, M. H. \& Sutherland, I. W. - Carbo. Polym., 3, p.23 (1983). http://dx.doi. org/10.1016/0144-8617(83)90010-3

6. Xu, X; Liu, W. \& Zhang, L. - Food Hydrocol., 20, p.723 (2006). http://dx.doi.org/10.1016/j.foodhyd.2005.06.012

7. Vendruscolo, C. T. - "Produção e caracterização do biopolímero produzido por Beijerinckia sp. isolada do solo da região de Ribeirão Preto-SP Brasil", Tese de Doutorado, Universidade Estadual de Campinas, Brasil (1995).

8. Moreira, A. S.; Vendruscolo, J. L. S.; Gil-Turnes, C. \& Vendruscolo, C. T. - Food Hydrocol., 15, p.469 (2001). http://dx.doi.org/10.1016/S0268-005X(01)00092-3

9. Pinto, E. P.; Moreira, A. S. \& Vendruscolo, C. T. - Rev. Bras. de Agroc., 8, p.247 (2002).

10. Antunes, A. E. C.; Moreira, A. S.; Vendruscolo, J. L. S \& Vendruscolo, C. T. - Braz. J. Food Technol., 6, p.317 ( 2003).

11. Borges, C. D.; Moreira, A. N.; Moreira, A. S.; Del Pino, F. A. B \& Vendruscolo, C. T. - Ciên. Tecnol. Alimentos, 24, 
p.327 (2004). http://dx.doi.org/10.1590/S010120612004000300004

12. Teixeira, A. M. - "Tempo de fermentação na produção $e$ qualidade de xantana e no perfil eletroforético de $X$. campestris $p v$ pruni", Dissertação de Mestrado, Universidade Federal de Pelotas, Brasil (2005).

13. Pinto, E. P. - "Desacetilação de xantana: influência no comportamento reológico", Dissertação de Mestrado, Universidade Federal de Pelotas, Brasil (2005).

14. Moreira, A. N.; Moreira, A. S.; Diaz, P. S.; Vendruscolo, C. T. \& Del Pino, F. A. B. - Braz. J. Food Technol., 8, p.135 (2005).

15. Borges, C. D. \& Vendruscolo, C. T. - Biosc. J., 23, p.67 (2007).

16. Klaic, P. M. A.; Nunes, A. M.; Moreira, A. S.; Vendruscolo, C. T. \& Ribeiro, A. S. - Carbo. Polym., 83, p.1895 (2011). http://dx.doi.org/10.1016/j.carbpol.2010.10.059

17. World Intellectual Property Organization - International Bureau. - "WO 2006/047845 A1 - Xanthan biopolymer, process and uses of the same", International publication date, 11 may (2006). Disponível em: <http://ww3.unit.br/ mestrados/biotecnologia>.

18. Instituto Nacional da Propriedade Industrial - INPI. - "Processo de produção de polissacarídeo por Beijerinckia sp", no PI 0105856-8, 07 nov. (2001). Disponível em: <http://pesquisa.inpi.gov.br/>.

19. McComb, E. A. \& McReady, R. M. Determination of acetyl in pectin and in acetylated carbohydrate polymers. Anal. Chem., 29, p.819 (1957). http://dx.doi.org/10.1021/ ac60125a025

20. Sloneker, J. H. \& Jeanes, A. - Canad. J. Chem., 40, p.2066 (1962). http://dx.doi.org/10.1139/v62-317

21. American Society for Testing and Materials - ASTM. - "D1428-64", Annual Book of ASTM Standards, Philadelphia (1981).

22. Petrobras. - "Norma Técnica N-2605 A - Ensaio de viscosificante para fluidos usados na exploração e produção de poços de petróleo e gás” (2003).

23. Burdock, G. A. - "Encyclopedia of Food and Color Additives”, CRC Press, New York (1997).

24. Food and Agriculture Organization of the United Nations - FAO. "Compendium of food additives specifications", addendum 7, FAO/WHO (1999).

25. Shatwell, K. P.; Sutherland, I. W. \& Ross-Murphy, S. B. - Int. J. Biol. Macromol., 12, p.71 (1990). http://dx.doi. org/10.1016/0141-8130(90)90056-G

26. Mariuzzo, D. M. - "Contribuição ao estudo da estrutura química do exopolissacarídeo PS-32 obtido por fermentação aeróbica da bactéria Beijerinkia sp", Dissertação de Mestrado, Universidade Estadual de Campinas, Brasil (1996).

27. Ohtani, K.; Yasuda, S.; Hiruta, O.; Uotani, K.; Tatsuta, K. \& Misaki, A. - Biosc. Biotechnol. Biochem., 59, p.1628 (1995). http://dx.doi.org/10.1271/bbb.59.1628
28. Cooke, A. A. \& Percival, E. - Carbo. Res., 43, p.117 (1975). http://dx.doi.org/10.1016/S0008-6215(00)83978-6

29. Sutherland, I. W. - Int. Dairy J., 11, p.663 (2001). http:// dx.doi.org/10.1016/S0958-6946(01)00112-1

30. Wang, F.; Wang, Y. \& Sun, Z. - J. Food Sci., 67, p.3289 (2002). http://dx.doi.org/10.1111/j.1365-2621.2002. tb09580.x

31. Goycoolea, F. M.; Milas, M. \& Rinaudo, M. - Int. J. Biol. Macromol., 29, p.181 (2001). http://dx.doi.org/10.1016/ S0141-8130(01)00164-7

32. Smith, I. H.; Symes, K. C.; Lawson, C. J. \& Morris, E. R. - Int. J. Biol. Macromol, 3, p.129 (1983). http://dx.doi. org/10.1016/0141-8130(81)90078-7

33. Ferreira, M. V. D.; Martins, A. L.; Costa, F. G.; Lopes, L. M.; Figueiredo, M. S.; Paula, B. R. F. C. \& Naccache, M. F. - "Evaluation of Polymers with Drag Reduction Potential", in: Anais do 2nd Mercosur Congress on Chemical Engineering and 4th Mercosur Congress on Process Systems Engineering, Enpromer CD-ROM, Rio de Janeiro - RJ, ago. (2006).

34. García-Ochôa, F.; Santos, V. E.; Casas, J. A. \& Gómez, E. - Biotechnol. Adv., 18, p.549 (2000). PMid: 14538095.

35. Pelletier, E.; Viebke, C.; Meadow, S. J. \& Williams, P. A. - Biopol. 59, p.339 (2001). http://dx.doi.org/10.1002/10970282(20011015)59:5<339::AID-BIP1031>3.0.CO;2-A

36. Sandford, P. A. \& Baird, J. - "Industrial utilization of polysaccharides", in: The Polysaccharide, S. O. Aspinall (Ed.), Academic Press, New York (1983).

37. Cadmus, M. C.; Knuston, C. A.; Lagoda, A. A.; Pittsley, J. E. \& Burton, K. A. - Biotechnol. Bioengin., 20, p.1003 (1978). http://dx.doi.org/10.1002/bit.260200703

38. Silveira, C. F.; Preichardt, L. D.; Moreira, A. S. \& Vendruscolo, C. T. - Rev. Bras. Tecnol. Agroind., 2, p.32 (2008). (1999).

39. Shah, A. K. \& Ashtaputre, A. A. - J. Indus. Microb. Biotechnol., 23, p.442 (1999).

40. Martínez-Padilla, L. P. \& Cabada-Celis, N. - Food Sci. Technol., 2, p.405 (1996). http://dx.doi. org/10.1177/108201329600200607

41. Boza, Y.; Neto, L. P.; Costa, F. A. A. \& Scamparini, A. R. P. - Proc. Biochem., 39, p.1201 (2004). http://dx.doi. org/10.1016/S0032-9592(03)00250-4

42. Petrobras. - "Norma Técnica N-2604 A - Viscosificante para fluidos usados na exploração e produção de poços de petróleo e gás" (2003).

43. Brasil. Agência nacional de Vigilância Sanitária - ANVISA. - "Resolução - RDC n 45, Regulamento técnico sobre aditivos alimentares autorizados para uso segundo as boas práticas de fabricação”, Diário Oficial da República Federativa do Brasil, Brasília (2010).

Enviado: $23 / 07 / 12$

Reenviado: $22 / 06 / 12$ Aceito: $23 / 07 / 12$ 